\section{Accelerated Oxidation of Cotton Cellulose in Presence of Reduced Cibanone Orange $\mathbf{R}$}

THE rate of oxidation of cellulose by dilute solutions of sodium hypochlorite has been shown to be accelerated if certain vat dyes are also present in the reduced form upon the fibre ${ }^{1,2}$. Ferrous hydroxide precipitated on cellulose exerts a similar influence on the rate of oxidation ${ }^{3}$. The mechanism of this acceleration is not yet clear. It has been suggested ${ }^{2}$ that the difference between the oxidation potential of the oxidizing agent and the reduction potential of the vat dye may be the determining factor in the acceleration of the oxidation. The present investigation deals with the measurement of reduction potentials of vat dyes upon the fibre and their influence on the acceleration of the rate of oxidation of the cellulose substrate by dilute sodium hypochlorite solutions.

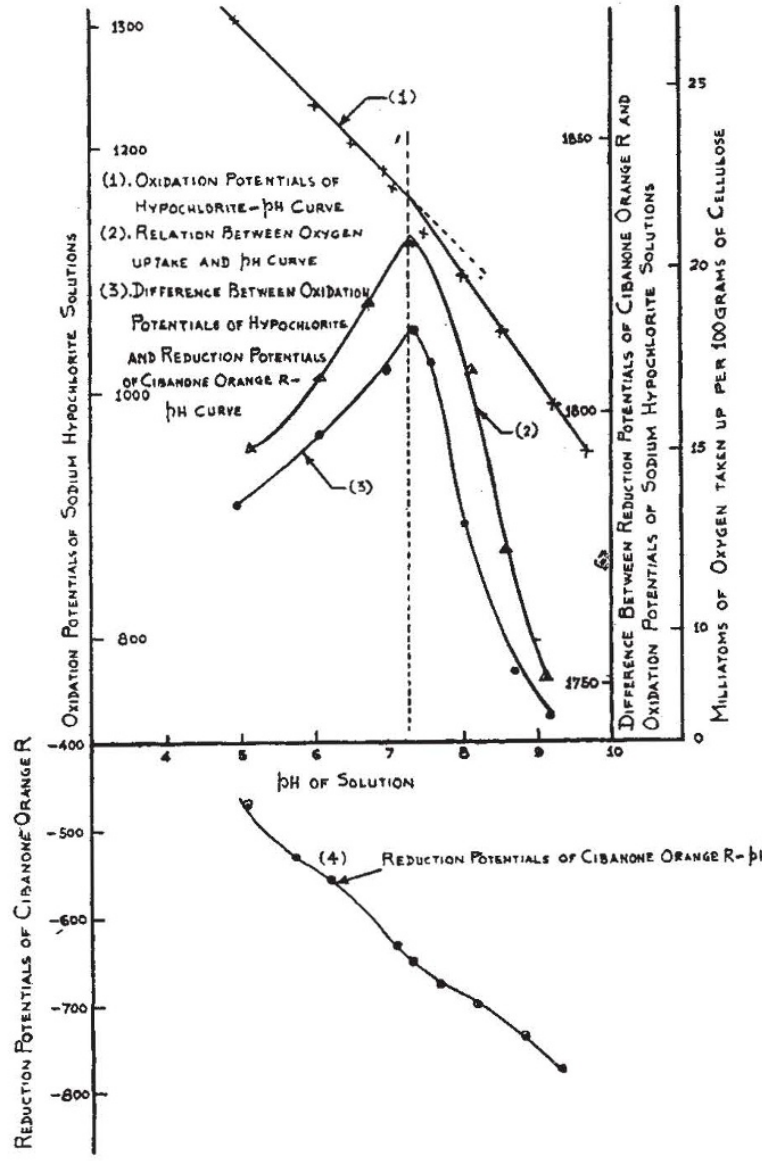

Loose cotton dyed with Cibanone Orange $\mathbf{R}$ was tightly wrapped round a stout platinum wire. The dyestuff on the cotton was chemically reduced, and the free leuco compound was prepared by washing away the soluble impurities with oxygen-free distilled water. The electrode was then immersed in an oxygen-free buffer solution of known hydrogen ion concentration through which hydrogen gas was bubbled vigorously for fifteen minutes. The E.M.F. of the following cell was measured at short intervals :
$+\mathrm{Hg} \mid \mathrm{Hg}_{2} \mathrm{Cl}_{2}$,Sat.KCl || Sat.KCl || Leuco Cibanone Orange $\mathrm{R}$ dyed cotton in buffer solution | Pt -

The E.M.F. reached a constant value after about fifteen to twenty minutes and resmained steady for periods varying between half to one hour. This constant value was taken as the E.M.F. of the cell. The potential of the half-cell

Pt | leuco Cibanone Orange $\mathrm{R}$ (dyed cotton in a buffer solution)

was calculated from the E.M.F. of the above cell. This value has been referred to as the reduction potential of Cibanone Orange R. The oxidation potentials of sodium hypochlorite solutions and the oxygen uptake by reduced Cibanone Orange $\mathbf{R}$ dyed cotton were measured by the method described by Nabar, Scholefield and Turner ${ }^{2}$. All measurements have been carried out at $30^{\circ} \mathrm{C}$. The results are illustrated in the accompanying graphs.

It will be noted that the curve relating $p \mathrm{H}$ to oxygen uptake closely resembles the curve showing the relation between the $p H$ and the difference between the oxidation potentials of hypochlorite solutions and the reduction potentials of Cibanone Orange R. A few other dyes examined show similar behaviour.

G. D. Joshi

G. M. NABAR

Department of Chemical Technology,

University of Bombay, Bombay 19.

Jan. 7.

1 Turner, Nabar and Scholefleld, J. Soc. Dyers and Col., 51, 5 (1935). 2 Nabar, Scholefleld and Turner, J. Soc. Dyers and Col.. 53, 5 (1937).

${ }^{3}$ Nabar and Turner, J. Soc. Dyers and Col., 61, 258 (1945).

\section{Effect of Alkaloids on Hydrogen Overpotential}

It is well known that certain substances, such as arsenious oxide ${ }^{1}$, when added to a solution in which is a working electrode evolving hydrogen, considerably increase the hydrogen overpotential at the cathode. Such substances are often referred to as 'poisons', and a detailed knowledge of their effects on irreversible electrode potentials appears to be of considerable importance in the elucidation of the mechanism of overpotential. It seemed probable that some additives might exist which function as 'activators' and thus decrease hydrogen overpotential at various current densities. Such substances have been found among certain alkaloids of the quinoline group.

Hydrogen overpotential has been measured by the direct method at current densities from $10^{-3}$ to $10^{-1}$ amp./sq.cm. on cathodes of mercury, lead, tungsten and platinized platinum in pure aqueous solutions of hydrogen chloride and in solutions containing quinoline, cinchonine, quinine, narcotine and morphine in concentrations from 2 to 150 micro-mol./litre. Marked lowering effects are observed in solutions containing these quantities of the alkaloids named above, particularly at lower current densities on merctury cathodes. Smaller lowering effects occur for lead; tungsten cathodes are little affected, and on platinized platinum all the above-mentioned alkaloids act as poisons. 